\title{
The Floppy Iris Syndrome - What Urologists and Ophthalmologists Need to Know
}

\author{
Faruquz Zaman Christian Bach Islam Junaid Athanasios G. Papatsoris \\ Jhumur Pati Junaid Masood Noor Buchholz \\ Department of Urology, Barts and the London NHS Trust, London, UK
}

\begin{abstract}
Key Words
Floppy iris syndrome • Cataract complication • Tamsulosin • Benign prostatic hyperplasia • Alpha adrenergic blocker
\end{abstract}

\begin{abstract}
Introduction: Benign prostatic hyperplasia (BPH) and cataract formation are common in older people. Medical management of symptomatic BPH is often preferred to surgical treatment as surgery increases the risk of morbidities, whereas, surgery is the main form of treatment to restore sight in patient with cataract. The clinical treatment of $\mathrm{BPH}$ is either alpha-1 adrenergic antagonist alone or combination of alpha reductase inhibitor and alpha adrenergic receptor (AR) antagonist. There are four alpha-AR antagonists currently available to treat $\mathrm{BPH}$. The uroselective alpha-blocker tamsulosin is the most commonly used drug among all. Studies showed that the majority of the patients who develop intraoperative floppy iris syndrome (IFIS) were on tamsulosin. Women are more likely to develop cataract than men and some recent studies showed that tamsulosin is effective in treating female lower urinary tract symptoms and thereby can cause IFIS during cataract surgery. Evidence Acquisition: We performed a critical review of the published articles and abstracts on association of IFIS with alpha-blockers and other medications as well as other medical conditions. Evidence Synthesis: Tamsulosin is the most common cause of formation of IFIS. However, not all patients given tamsulosin develop IFIS and cases have been reported without any tamsulosin treatment. Conclusion: Tamsulosin is a recognized
\end{abstract}

\section{KARGER}

Fax +4161306 1234

E-Mail karger@karger.ch

www.karger.com
(C) 2012 S. Karger AG, Basel

1015-9770/12/0061-0001\$26.00/0

Accessible online at:

www.karger.com/cur cause to impede mydriasis and lead to IFIS during cataract surgery. Urologist should collaborate with their ophthalmology colleagues and general practitioner during prescribing tamsulosin in patients with history of cataract or waiting for planned cataract surgery. The increasing life expectancy and growth of older people will increase the number of men and women who suffer from lower urinary tract symptoms as well as cataract. Therefore, further research and studies are required to properly understand the relation of alpha blockers and IFIS.

Copyright $\odot 2012$ S. Karger AG, Basel

\section{Introduction}

The intra-operative floppy iris syndrome (IFIS) is a variant of the small pupil syndrome. It has been observed during cataract surgery in some patients currently or previously treated with the $\alpha 1$ adrenoceptor (AR) antagonist tamsulosin. IFIS was first described by Chang et al. [1] in 2005. These patients tend to dilate poorly and to respond poorly to stretching during surgery. Without adequate pupil dilatation, IFIS may shrink the visualisation of the surgical field, including the cataract itself. This may impair removal of cataract and can lead to other complications such as rupture of the posterior capsule, which further increases the risk of other vision-threaten- 
ing complications of cataract surgery [2]. Isolated cases of IFIS have been observed with other AR antagonists (alfuzosin and doxazosin), but to a lesser extent than for tamsulosin. However, reports have also been found in the absence of tamsulosin. IFIS is characterised by loss of muscle tone in the iris with symptom triad of pupil constriction despite pre-operative dilatation with standard mydriatic drugs, fluttering and bellowing of iris stroma, and a marked tendency for the iris to prolapse towards the side port incisions [1]. Ohtake et al. [3] showed that tamsulosin and other AR antagonists inhibit phenylephrine induced mydriasis and cause miosis to an equal extent and duration in an animal study.

\section{Discussion}

We know that age is a predominant risk factor for both benign prostatic hyperplasia (BPH) and cataract. Physicians can expect to see an increasing numbers of aging males on $\alpha 1 \mathrm{AR}$ antagonists who require operative intervention for cataract removal as $\alpha 1$ AR blocker is the most common drug for BPH. The other commonly used medication for BPH is 5 alpha reductase inhibitors (5ARIs) which alleviates lower urinary tract symptoms by reducing the size of prostate over a period of a few months. There are two 5ARIs available for the treatment of BPH: finasteride and dutasteride. Finasteride inhibits only the type 2 isoenzyme of 5 alpha-reductase, whereas dutasteride blocks both, type 1 and type 2 isoenzyme of 5 alpha-reductase and thereby prevents conversion of testosterone to dihydrotestosterone more completely.

Patients taking $\alpha 1$ AR antagonists may be at risk of developing IFIS during cataract surgery. Recently urologists have begun to use tamsulosin to treat lower urinary tract symptoms in women as well. Therefore, ophthalmologists could expect to encounter this surgical problem more frequently with increasing use of this drug in the elderly female population that also require cataract surgery [4].

Although the precise mechanism by which tamsulosin can lead to IFIS remains unknown, Chang et al. [1] suggest that tamsulosin has a high affinity and specificity for the $\alpha 1_{\mathrm{A}}$ adrenergic receptor, which is thought to be the dominant receptor in the iris. They discussed data from one retrospective and another prospective study, which support an association between tamsulosin and IFIS. The retrospective study assessed 706 eyes in 511 patients who underwent cataract surgery, 27 (5\%) of whom were receiving systemic $\alpha 1$ adrenoceptor antagonists (16 tam- sulosin, and 11 prazosin, terazosin, or doxazosin). The researchers noted a so-called floppy iris in 10 of the 16 patients given tamsulosin, and recorded poor or moderately poor preoperative dilation in patients taking prazosin, terazosin, or doxazosin (but no actual cases of IFIS were identified). In the prospective study, IFIS was diagnosed in 21 of 900 cataract surgeries (741 patients), and in 16 of 741 patients (about $2 \%$ ). Fourteen of the 16 patients had documented concomitant use of tamsulosin at the time of cataract surgery, and 1 patient had stopped tamsulosin 3 year prior to surgery; 1 patient had no history of tamsulosin exposure. Moreover, IFIS was reported in both eyes of the 5 patients taking tamsulosin who required bilateral cataract operation. Of the 725 patients who did not have IFIS none were receiving tamsulosin. Frequency of IFIS in both studies, totalling more than 1,600 eyes and 1,250 patients, was reported as $2 \%$ of patients having cataract surgery, and was regarded as associated with tamsulosin. Moreover, roles have been suggested for the serotoninergic and dopaminergic receptors and of the $1_{L}$ subtype of the $\alpha$-receptor.

In another prospective observational study conducted in a district general hospital in the UK, Amin K et al. [5] analysed a total of 1,462 cataract cases (1,267 patients) performed over 6 months. Of these, 23 eyes of 16 patients who were on tamsulosin were recruited into the study. All the patients were men with the mean age of 76 years. A small pupil was demonstrated intra-operatively in 69\% (16/23) of the eyes. A floppy iris or iris prolapse during surgery was reported on $57 \%$ (13/23) of the eyes. Complications were posterior capsule rupture in 1 case $(4 \%)$ and iris trauma in 5 cases $(22 \%)$. The incidence of IFIS in patients undergoing cataract surgery over 6 months has been calculated therefore $0.9 \%$ in a representative UK patient population. It seems that IFIS have a strong association with tamsulosin use.

More recently, IFIS was reported to be $1.6 \%$ in a study population of 774 patients [6]. IFIS was documented in 14 of 18 patients $(77.8 \%)$ taking tamsulosin. Consistent with the original report by Chang et al. [1], tamsulosin use overall was observed in $2.2 \%$ of patients in the age group prone for cataract.

Some studies have attempted to quantify the risk of IFIS with tamsulosin and to clarify the role of other $\alpha 1$ adrenergic receptor antagonists in IFIS. In a UK-based observational prospective study of 2,390 cataract procedures, Cheung et al. [7] identified 3 eyes with pure IFIS and 6 eyes with some features of IFIS out of 15 patients (17 eyes) given tamsulosin. Notably, the duration of tamsulosin did not correlate with the severity of IFIS. 
Table 1. Comparison of effects of tamsulosin and other alpha-blockers

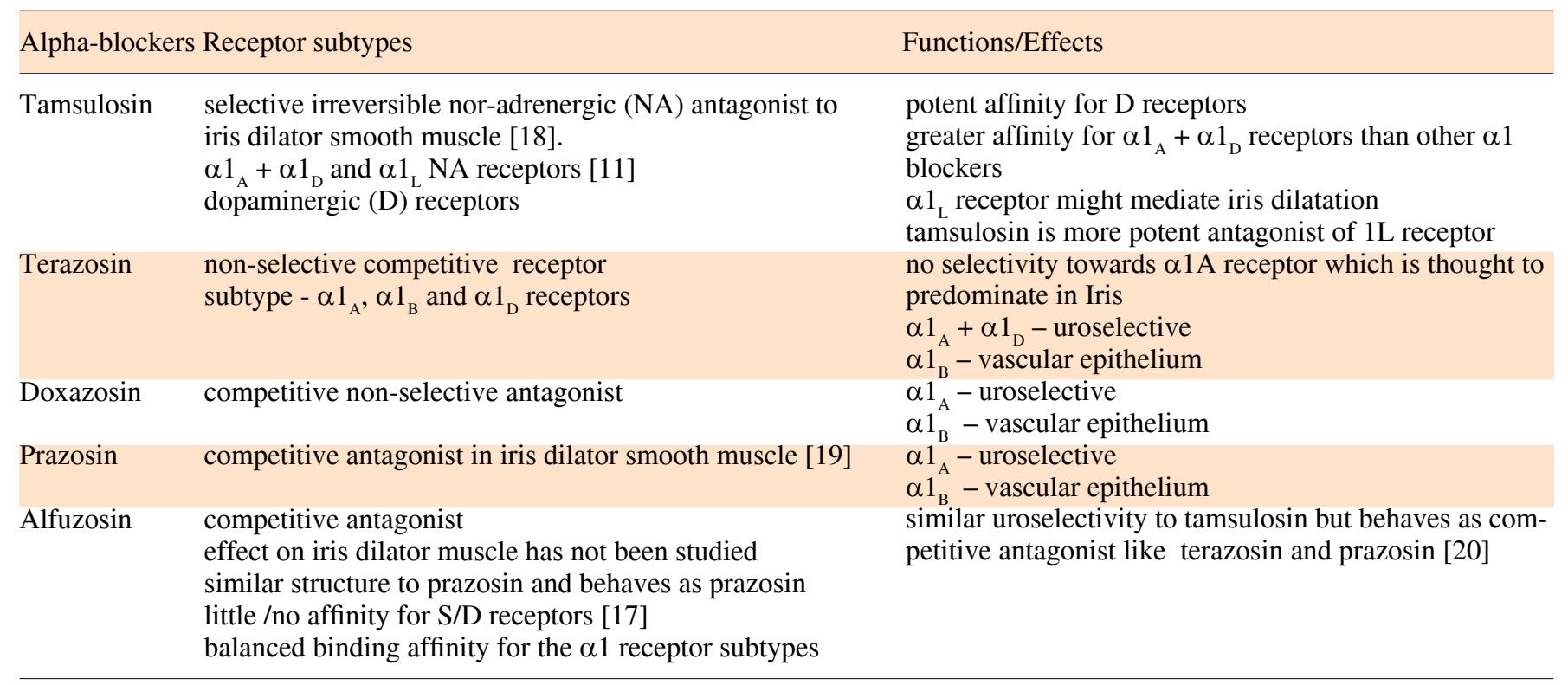

\section{Pathophysiology of IFIS}

The most comprehensive review of adrenergic receptors in relation to the potential pathophysilogy of IFIS was recently published [8]. Contraction of the iris dilator muscle via adrenergic stimulation results in mydriasis, which is necessary during cataract surgery. Therefore, agents such as topical phenylephrine, a $\alpha 1$ AR agonist, are routinely used in cataract surgery. Besides the effect of $\alpha 1 \mathrm{~A} \mathrm{AR}$ on prostate tissue, several animal studies have isolated the $\alpha 1 \mathrm{~A}$ AR subtype as the mediator of iris smooth muscle dilatation [9]. It has been predicted that since tamsulosin is the only specific $\alpha 1_{\mathrm{A}} \mathrm{AR}$ antagonist marketed for $\mathrm{BPH}$, it may also inhibit $\alpha 1_{\mathrm{A}}$ receptor in the iris, thereby leading to IFIS. There are 3 subtypes of $\alpha 1$ ARs $-\alpha 1_{A}, \alpha 1_{B}$ and $\alpha 1_{D}$ have all been cloned and characterised [10]. A fourth subtype $\alpha 1_{L}$ AR has been pharmacologically defined but not fully characterised or cloned. Tiwari et al. [11] have also proposed an important role for the $\alpha 1_{L}$ subtype receptor in mediation of iris dilatation in the human eye, and tamsulosin is considered to be a more potent antagonist of this receptor subtype than terazosin or doxazosin. As the exact mechanism by which tamsulosin induces IFIS has yet to be established, the role of other non-adrenergic receptors (i.e. dopaminergic and serotonergic receptors) in inducing pupillary changes warrants consideration [12-16]. Tamsulosin seems to have a potent affinity for dopaminergic receptors as well [17].

Table 1 compares the mechanisms and effects of tamsulosin and other AR antagonists.

\section{Evidence Acquisition}

We performed a critical review of the published articles and abstracts on association of IFIS with alphablockers and other medications as well as other medical conditions.

\section{Evidence Synthesis}

\section{Patients on Tamsulosin before Cataract Operation}

In humans, tamsulosin is an irreversible antagonist of $\alpha 1$ adrenoceptors, and thus a withdrawal period of several days may not be sufficient to suppress the blockade of $\alpha 1$ adrenoceptors. There is little research between the length of time of tamsulosin use and the development of IFIS. Chang et al. [1] found that discontinuation of tamsulosin 4-7 days before surgery was helpful, but did not prevent IFIS completely. Settas et al. [21] reported no benefit from temporarily stopping alfuzosin treatment 
before surgery. Discontinuation of tamsulosin for 1 week or less before surgery might be insufficient because the elimination half-life for tamsulosin is about $48-72$ hour. Chang et al. [1] also suggested that IFIS might be semipermanent, and that blockade of $\alpha 1_{\mathrm{A}}$ receptors in the iris dilator muscle could result in disuse atrophy of the muscle because IFIS was still evident in some patients despite drug withdrawal. Cheung et al. [7] postulates that if the proposed mechanism of disuse atrophy is correct one should expect a minimum time tamsulosin use before IFIS develops. In their prospective study looking at the prevalence of IFIS in patients taking tamsulosin, they noted that of the 17 eyes studied the shortest duration of tamsulosin use associated with IFIS was 3 months. However, Taben et al. [22] reported a case of IFIS after 1 day of tamsulosin (1 dose) therapy in patient with tamsulosin which may mean alpha 1 blockade alone without disuse atrophy could be enough to cause IFIS. Shah et al. [23] also published 1 case of IFIS occurring only 2 days after tamsulosin was started. Tamsulosin is often prescribed as a short term pharmacologic adjunct for the treatment of ureteric calculus. This is relevant because it appears that tamsulosin can cause IFIS almost immediately. In other patients however, pre-operative dilatation and iris floppiness seemed to improve after drug withdrawal. Chang et al. [1] recommended temporary withdrawal of tamsulosin treatment 2 weeks before surgery. Michel et al. [24] have assessed the effects of $\alpha 1$ adrenergic receptor antagonists on pupil size and intraurethral pressure, and Schwinn et al. $[25,26]$ have depicted the effect of therapeutic blood level of any $\alpha 1$ adrenergic receptor antagonist given up to the day surgery. They advised that it would be prudent to stop all $\alpha 1$ adrenergic receptor antagonists before cataract surgery to avoid the possibility of IFIS, although the exact time and effectiveness of this action still need to be determined by careful recording of drug doses and plasma levels in future prospective studies.

Stoppage of tamsulosin 1-2 weeks prior to cataract surgery is anecdotally considered helpful, but the benefit and the duration of requirement of discontinuing the therapy prior to cataract surgery has not yet been established and remain unclear.

\section{Effects of Tamsulosin after Cataract Surgery}

There are only few studies which assessed the connection between tamsulosin exposure and post-operative complications. Scientists focused on 96,128 men aged 66 and older who underwent cataract surgery in Canada between 2002 and 2007. Of those, one group of 3,550 patients had taken tamsulosin while another 7,426 had taken different alpha blocking agent within 2 weeks of cataract surgery. In total 284 of the patients in the study experienced relevant side effects related to cataract surgery. The research found that patients who suffered complications were 2.3 times more likely to have taken tamsulosin than other alpha blockers, which showed no increased risk of eye injury. According to researchers [27], one in every 255 cataract surgery patients who take tamsulosin within 14 days of the surgery will develop severe eye complications and thereby increase the risk of needing second surgery. The authors also concluded that recent use of other alpha blockers and previous use of tamsulosin or other alpha-blockers had no significant effect on post-operative adverse events like retinal detachment, lost lens or lens fragment, or endophthalmitis.

On the other hand, Taben et al. [22] described in a case report that a patient had IFIS during cataract surgery 7 weeks after taking a single dose of tamsulosin. Eight months after tamsulosin use his pupils still dilated poorly, though slightly better than during surgery. This indicates that stopping the drug preoperatively may not decrease the incidence or severity of IFIS. Tamsulosin may bind $\alpha 1$ receptors on the iris longer than elsewhere. From an experimental study, it has been noted in Albino rabbits that IV alpha antagonists induced miosis was reversed 8 hours after cessation of the drug [24]. However, binding characteristics of alpha antagonists in human and darkly pigmented irises have not been well studied.

In contrast, Schwinn et al. $[25,26]$ considered the implications of withdrawing of $\alpha 1$-adrenergic receptor antagonist treatment and add that restarting of $\alpha 1$ adrenergic receptor antagonists immediately after cataract surgery seems carry little or no risk.

As the combination of cataract surgery and tamsulosin exposure is relatively common, patients should be properly informed of the risks of tamsulosin therapy, and during pre-operative assessments it is important to identify the use of tamsulosin by the patients as well as pre-operative assessment staffs.

\section{Comparison of Tamsulosin with other Alpha \\ Blockers in Relation to IFIS}

Structural differences and well-defined mechanisms of action of AR antagonists might account for substantial differences in cases of IFIS.

Alfuzosin has similar uroselectivity to tamsulosin and it inhibits completely and selectively $\alpha 1$ adrenergic receptors in the prostate, bladder base and prostatic urethra. Alfuzosin has been reported to show selectivity only for the $\alpha 1_{\mathrm{A}}$ subtype [28] and act as a pure competi- 
tive antagonist in human prostatic smooth muscle [29], as do terazosin [30] and prazosin [20]. Similar to prazosin [19], alfuzosin behaves as a competitive antagonist in human iris smooth muscle. Settas et al. [21] suggested that the overall affinity of $\alpha 1$ adrenoceptor antagonists towards $\alpha 1_{\mathrm{A}}$ receptors might explain IFIS.

Chang et al. [1] noted poor or moderately poor preoperative dilatation, but no definite cases of IFIS, in the group of patients taking prazosin, terazosin, and doxazosin. Evidence remains insufficient to suggest that IFIS is always a side effect of $\alpha 1$ adrenoceptor antagonists. Isolated cases of IFIS associated with other $\alpha 1$ adrenergic receptor antagonists have however been reported.

Another UK prospective study reported greater use of doxazosin $(n=11)$ in 100 patients awaiting cataract surgery than of any other $\alpha 1$ adrenoceptor antagonists, such as indoramin $(\mathrm{n}=8)$, prazosin $(\mathrm{n}=5)$, terazosin $(\mathrm{n}$ $=2)$, and tamsulosin $(\mathrm{n}=3)$. Of this unselected population, no patients developed IFIS and 1 had a constricted pupil [31].

A recent study directly compared the incidence of IFIS attributable to tamsulosin with an active comparator group [32]. In this retrospective study of 64 men totalling 92 eyes, there was an increased risk of IFIS in patients exposed to tamsulosin (86.4\%) when compared to alfuzosin $15.4 \%$. The adjusted odds ratio for IFIS in patients taking tamsulosin when compared to alfuzosin was $32.15 \%$ (95\% CI). Furthermore, a fivefold increase in surgical complication rates was observed in patients diagnosed with IFIS, highlighting its clinical significance.

Currently, the risk of IFIS has only been demonstrated with systemic use of $\alpha 1$ AR antagonists. In a study comparing the incidence of IFIS between topical and systemic use of $\alpha 1-A R$ antagonists, no cases were observed in patients taking bunazosin, a topical non-selective $\alpha 1 \mathrm{AR}$ antagonist [33].

\section{Relation of IFIS with $\alpha 1_{A}$ Antagonists and} Associated Diseases/Medications

Chadha et al. [34] assessed the analysed case of floppy iris during cataract surgery and the use of $\alpha 1$ adrenoceptor antagonists and the presence of diabetes mellitus in 1,786 patients $(1,842$ eyes). Eleven eyes in 11 patients had complete IFIS, and 18 eyes in 18 patients had incomplete IFIS; 12 eyes in 21 patients given tamsulosin had signs of complete or incomplete IFIS; however, 17 cases of IFIS ( 5 complete, none of whom had ever taken an $\alpha 1$ adrenoceptor antagonists, and 12 incomplete -1 of whom was receiving doxazosin) were noted for patients who were not given tamsulosin. None of the other pa-

Association of the Floppy Iris Syndrome and Alpha Adrenergic Antagonists tients taking doxazosin $(n=48)$, or those taking alfazosin $(n=2)$ or terazosin $(n=1)$ had signs of IFIS. No relation between diabetes and IFIS was found. The researchers conclude that non-selective $\alpha 1$ adrenoceptor antagonists are unlikely to be associated with IFIS, but they suggest that other factors apart from tamsulosin may play an important part.

In a case report Taben et al. [22] described that a patient with a history of dermatomyositis developed IFIS 1 day after a single dose of tamsulosin. Similar findings were noted intraoperatively 7 weeks later in the second eye after no further tamsulosin therapy. A single dose of tamsulosin and/or dermatomyositis may be associated with IFIS.

In addition, Schwinn et al. [26] proposed that IFIS may be associated with various diseases and medications. They mentioned that in animal studies, nitric oxide has been shown to relax iris sphincter and dilator smooth muscle. The patient who uses a nitro-glycerin patch daily could be another potential cause of IFIS. However, there are no clinical cases or human studies showing an association between nitrates and IFIS.

Furthermore, saw palmetto (Serona repens), a widely used alternative therapy for $\mathrm{BPH}$, was also associated with IFIS in 2 patients [35]. Neither of these two patients had taken prescription medications for BPH and they develop moderate IFIS. Despite the development of IFIS, the authors reported no significant surgical complications.

As we mentioned earlier, 5ARI, finasteride and dutasteride reduce prostate size by inhibiting conversion of testosterone to dihydrotestosterone, taking 6 months for a full therapeutic response. Two cases of IFIS were associated with finasteride intake [36]. Neither of the patients had taken systemic $\alpha 1$ AR antagonists prior to surgery, and to date these are the only published cases associated with 5ARI therapy.

There are some cases of IFIS have been linked to other medications including chlorpromazine, labetolol, and donepezil, which have three distinct mechanisms of action [37-39].

\section{Conclusion}

The $\alpha 1_{\mathrm{A}}$ adrenoceptor antagonist tamsulosin is recognised to impede mydriasis and increase the risk of IFIS. However, the exact mechanism remains under investigation. On one hand, not all patients given tamsulosin develop IFIS; on the other hand cases have been reported

Curr Urol 2012;6:1-7 
without any tamsulosin treatment prior to or after cataract surgery. Some patients with tamsulosin-related IFIS improve after drug withdrawal, whereas others keep having symptoms years after the treatment has stopped.

Patients should be educated and consented regarding potential risks of this drug class. The patient's ophthalmologist should be prepared for possible modifications of their surgical technique. The urology community should be aware of the potential risks and should collaborate with their ophthalmology colleagues and general practitioners (GP) during prescribing tamsulosin in patients with history of cataract or waiting for planned cataract surgery. The increasing life expectancy and growth of older people will increase the number of men and women who would potentially suffer from LUTS as well as cataract. Therefore, further research and studies are required to properly understand the relation of alpha blockers and IFIS.

\section{References}

1 Chang DF, Campbell JR: Intraoperative floppy iris syndrome associated with tamsulosin. J Cataract Refract Surg 2005;31:664-673.

-2 Schwinn DA, Afshari NA: alpha 1-adrenergic receptor antagonists and the iris: new mechanistic insights into floppy iris syndrome. Surv Ophthalmol 2006;51:501-512.

3 Ohtake A, Someya A, Watanabe M: Effects of tamsulosin and other a1-adrenoceptor antagonists on pupil size in rabbits. Astellas Study Report for Study PHA050007, 2005.

4 Reitz A, Haferkamp A, Kyburz T, Knapp PA, Wefer B, Schurch B: The effect of tamsulosin on the resting tone and the contractile behaviour of the female urethra: a functional urodynamic study in healthy women. Eur Urol 2004;46:235-240.

5 Amin K, Fong K, Horgan SE: Incidence of intra-operative floppy iris syndrome in a UK district general hospital and implications for future workload. Surgeons 2008;6:207-209.

6 Takmaz T, Can I: Clinical features, complications, and incidence of intraoperative floppy iris syndrome in patients taking tamsulosin. Eur J Ophthalmol 2007;17:909-913.

7 Cheung CM, Awan MA, Sandramouli S: Prevalence and clinical findings of tamsulosinassociated intraoperative floppy-iris syndrome. J Cataract Refract Surg 2006;32:1336-1339.

$>8$ Srinivasan S, Radomski S, Chung J, Plazker $\mathrm{T}$, Singer S, Slomovic AR: Intraoperative floppy-iris syndrome during cataract surgery in men using alpha-blockers for benign prostatic hypertrophy. J Cataract Refract Surg 2007;33:1826-1827.

$>9$ Marklund-Bau H, Edell-Gustafsson U, Spangberg A: Bothersome urinary symptoms and disease specific quality of life in patients with benign prostatic onstruction. Scand $\mathbf{J}$ Urol Nephrol 2007;41:32-41.

10 Hieble JP, Bylund DB, Clarke DE, Eikenburg DC, Langer SZ, Lefkowitz RJ, Minneman KP, Ruffolo RR Jr: International Union of Pharmacology. X. Recommendation for nomenclature of alpha 1-adrenoceptors: consensus update. Pharmacol Rev 1995;47:267-270.
11 Tiwari A: Tamsulosin and floppy iris syndrome in benign prostatic hyperplasia patients. Expert Opin Investig Drugs 2006;15: 443-446.

-12 Fanciullacci M, Sicuteri R, Alessandri M, Geppetti P: Buspirone, but not sumatriptan, induces miosis in humans: relevance for a serotoninergic pupil control. Clin Pharmacol Ther 1995;57:349-355.

13 Yu Y, Ramage AG, Koss MC: Pharmacological studies of 8-OH-DPAT-induced papillary dilation in anesthetized rats. Eur J Pharmacol 2004;489:207-213.

14 Pringle E, Packard R: Antipsychotic agent as an etiologic agent of IFIS. J Cataract Refract Surg 2005;31:2240-2241.

15 Giuliano F: Impact of medical treatments for benign prostatic hyperplasia on sexual function. BJU Int 2006;97(suppl 2):34-38.

16 Clement P, Bernabe J, Kia HK, Alexandre L, Giuliano F: D2-like receptors mediate the expulsion phase of ejaculation elicited by 8-hydroxy-2-(di-N-propylamino) tetralin in rats. J Pharmacol Exp Ther 2006;316:830-834.

17 Leonardi A, Hieble JP, Guarneri L, Naselsky DP, Poggesi E, Sironi G, Sulpizio AC, Testa $\mathrm{R}$ : Pharmacological characterization of uroselective alpha-1 antagonist Rec 15/2739 (SB 216469): role of the alpha-1L adrenoceptor in tissue selectivity, part 1. J Pharmacol Exp Ther 1997;281:1272-1283.

18 Takayanagi I, Konno F, Kameda H, Kubo H, Furukawa A, Toyoda T: A difference in mode of antagonism between optical isomers of a potent selective alpha 1-adrenoceptor blocker (YM-12617) and norepinephrine in isolated rabbit iris dilator and aorta. Jpn J Pharmacol 1986;42:579-582.

19 Ishikawa H, Miller DD, Patil PN: Comparison of post-junctional alpha-adrenoceptors in iris dilator muscle of humans, and albino and pigmented rabbits. Naunyn Schmiedebergs Arch Pharmacol 1996;354:765-772.

20 Teng CM, Guh JH, Ko FN: Functional identification of alpha 1-adrenoceptor subtypes in human prostate: comparision with those in rat vas deferens and spleen. Eur J Pharmacol 1994;265:61-66.
21 Settas G, Fitt AW: Intraoperative floppy iris syndrome in a patient taking alfuzosin for benign prostatic hyperplasia. Eye (London) 2006;20:1431-1432.

22 Taben R, Thakuria P, Jones M, Lohman LE: Intraoperative floppy-iris syndrome after one day of tamsulosin therapy. http:// stream.expoplanner.com/ascrs2007/powerpoints/000092_IFIS_Case_ASCRS_Presentation.ppt

23 Shah N, Tendulkar M, Brown R: Should we anticipate intra-operative floppy iris syndrome (IFIS) even with very short history of tamsulosin? Eye (London) 2009;23:740.

24 Michel MC, Okutsu H, Noguchi Y, Suzuki M, Ohtake A, Yuyama H, Yanai-Inamura H, Ukai M, Watanabe M, Someya A, Sasamata M: In vivo studies on the effects of alpha 1adrenoceptor antagonists on pupil diameter and urethral tone in rabbits. Naunyn Schmiedebergs Arch Pharmacol 2006;372:346-353.

25 Schwinn DA, Afshari NA: Alpha1-adrenergic antagonists and floppy iris syndrome: tip of the iceberg? Ophthalmology 2005;112: 2059-2060.

26 Schwinn DA, Afshari NA: Alpha(1)-adrenergic receptor antagonists and the iris: new mechanistic insights into floppy iris syndrome. Surv Ophthalmol 2006;51:501-512.

27 Bell CM, Hatch WV, Fischer HD, Cernat G, Paterson JM, Gruneir A, Gill SS, Bronskill SE, Anderson GM, Rochon PA: Association between tamsulosin and serious ophthalmic adverse events in older men following cataract surgery. JAMA 2009;301:1991-1996.

$\checkmark 28$ Martin DJ, Angel I, Arbilla S: Functional uroselectivity. Eur Urol 1998;33(suppl 2):12-18.

29 Palea S, Barras M: Antagonist effects of alfuzosin on concentration-response curves to phenylephrine and noradrenaline in human prostatic adenoma (abstract). Neurourol Urodyn 2000;19:431-433.

30 Muramatsu I, Taniguchi T, Okada K: Tamsulosin: alpha1-adrenoceptor subtype-selectivity and comparison with terazosin. Jpn J Pharmacol 1998;78:331-335. 
-31 Parmar B, Qatarneh D, Claoue C: Alpha antagonists in cataract surgery. J Cataract Refract Surg 2005;31:2241.

32 Blouin MC, Blouin J, Perreault S, Lapointe A, Dragomir A: Intraoperative floppy-iris syndrome associated with alpha 1-adrenoceptors: comparison of tamsulosin and alfuzosin. J Cataract Refract Surg 2007;33:1227-1234.

- 33 Oshika T, Ohashi Y, Inamura M, Ohki K, Okamoto S, Koyama T, Sakabe I, Takahashi K, Fujita Y, Miyoshi T, Yasuma T: Incidence of intraoperative floppy iris syndrome in patients on either systemic or topical alpha(1)adrenoceptor antagonists. Am J Ophthalmol 2007;143:150-151.
34 Chadha V, Borooah S, Tey A, Styles C, Singh J: Floppy iris behaviour during cataract surgery: associations and variations. $\mathrm{Br} \mathrm{J}$ Ophthalmol 2007;91:40-42.

35 Yeu E, Grostern R: Saw palmetto and intraoperative floppy-iris syndrome. J Ctaract Refract Surg 2007;33:927-928.

36 Issa SA, Dagres E: Intraoperative floppy-iris syndrome and finasteride intake. J Cataract Refract Surg 2007;33:2142-2143.
37 Unal M, Yucel I, Tenlik A: Intraoperative floppy-iris syndrome associated with chronic use of chlorpromazine. Eye (London) 2007; 21:1241-1242.

38 Calotti F, Steen D: Labetalol causing intraoperating floppy-iris syndrome. J Cataract Refract Surg 2007;33:170-171.

39 Papadopoulos R, Bachariou A: Intraoperative floppy-iris syndrome associated with chronic intake of donepezil. J Cataract Refract Surg 2007;33:1997-1998. 